\title{
Sind komplementärmedizinische Behandlungen preiswerter?
}

\author{
Studer H-P, Busato A: Comparison of Swiss Basic Health Insurance Costs of Complementary and \\ Conventional Medicine. Forsch Komplementmed 2011;18:315-320.
}

\begin{abstract}
Background: From 1999 to 2005, 5 methods of complementary and alternative medicine (CAM) applied by physicians were provisionally included into mandatory Swiss basic health insurance. Between 2012 and 2017, this will be the case again. Within this process, an evaluation of cost-effectiveness is required. The goal of this study is to compare practice costs of physicians applying CAM with those of physicians applying solely conventional medicine (COM). Methods: The study was designed as a cross-sectional investigation of claims data of mandatory health insurance. For the years 2002 and 2003 , practice costs of 562 primary care physicians with and without a certificate for CAM were analyzed and compared with patient-reported outcomes. Linear models were used to obtain estimates of practice costs controlling for different patient populations and structural characteristics of practices across CAM and COM. Results: Statistical procedures show similar total practice costs for CAM and COM, with the exception of homeopathy with $15.4 \%$ lower costs than COM. Furthermore, there were significant differences between CAM and COM in cost structure especially for the ratio between costs for consultations and costs for medication at the expense of basic health insurance. Patients reported better quality of the patient-physician relationship and fewer adverse side effects in CAM; higher cost-effectiveness for CAM can be deduced from this perspective. Conclusion: This study uses a health system perspective and demonstrates at least equal or better cost-effectiveness of CAM in the setting of Swiss ambulatory care. CAM can therefore be seen as a valid complement to COM within Swiss health care.
\end{abstract}

\section{Kommentar}

Es wird immer wieder behauptet, dass komplementärmedizinische Behandlungen preisgünstiger seien als konventionelle Medizin (COM). Wirklich aussagekräftige Studien hierzu sind jedoch rar. Studer und Busato werteten Daten aus, die 2002/2003 im Rahmen des PEK(Programm Evaluation Komplementärmedizin)-Projekts erhoben wurden. Hierbei handelt es sich um eine Querschnittserhebung von Ärzten, deren Patientendaten gesammelt wurden, insbesondere erstattete Kosten, aufgeschlüsselt nach Konsultationskosten, Kosten für diagnostische und therapeutische Massnahmen, für dispensierte oder verschriebene Medikamente sowie für externe Laborkosten und Physiotherapie.

Nach Ausschluss von Anbietern mit geringem Umsatz unter 30000 CHF wurden 562 Ärzte in die Auswertung einbezogen und wie folgt zugeordnet: 173 COM-Anbieter, 220 zertifizierte Anbieter von Komplementär- und Alternativmedizin (CAM) mit Schwerpunkt auf ein Verfahren, 137 nichtzertifizierte CAM-Anbieter und 32 COM- und CAM-Anbieter mit mehreren Verfahren. Die Zertifizierungen lagen vor bei 102 homöopathischen Ärzten, gefolgt von 61 TCMzertifizierten und 26 anthroposophischen Ärzten, während Ärzte mit Spezialisierung auf Neuraltherapie (19) und Phytotherapie (12) deutlich seltener vorkamen. 32 Ärzte hatten mehrere CAM-Zertifizierungen.

In der Rohauswertung zeigen sich bereits Unterschiede hinsichtlich der Kosten bei den verschiedenen Ärztegruppen, aber auch deutlich jüngere Patienten und ein höherer Frauenanteil sowie etwas weniger Konsultationen pro Jahr insbesondere bei den Homöopathen im Vergleich zur COM. Weiterhin zeigen die Rohdaten deutlich geringere Kosten für Homöopathie-Behandlungen und anthroposophische Behandlungen im Vergleich zur COM, während Phytotherapie und Neuraltherapie dazwischen lie- gen. Dies mag aber daran liegen, dass sich die Patienten eben grundlegend unterscheiden.

Es wurde nun eine komplexe logistische Regression angewandt, um die Unterschiede in der Patientenstruktur auszugleichen. Danach sind die Homöopathie-Kosten nur noch 15\% geringer als COM, andere Verfahren wie Phytotherapie gleichwertig und TCM sogar «teurer» (um 5,4\%). Die einzelnen Kosten unterscheiden sich deutlicher: Neuraltherapie und TCM zeigen höhere Konsultationskosten und geringere Medikationskosten. Die Unterschiede sind bei TCM im Vergleich zur COM aber nur für Labor- und Physiotherapiekosten signifikant niedriger. Die Arzneikosten liegen bei der anthroposophischen Behandlung am höchsten (138\% von COM).

In der Diskussion wird die Möglichkeit offengelassen, dass Unterschiede in der Patientenstruktur nicht komplett auszugleichen waren. Dies ist ein Manko entsprechender Quer-

\begin{tabular}{|c|c|}
\hline KARGER & (c) 2013 S. Karger GmbH, Freiburg \\
\hline $\begin{array}{l}\text { Fax +49761 } 4520714 \\
\text { Information@Karger.com } \\
\text { www.karger.com }\end{array}$ & $\begin{array}{l}\text { Accessible online at: } \\
\text { www.karger.com/szg }\end{array}$ \\
\hline
\end{tabular}

Fax +497614520714

www.karger.com
Prof. Dr. med. Dr. rer. nat. Bernhard Uehleke

Institut für Naturheilkunde, Universitätsspital Zürich

Sonneggstrasse 6, 8006 Zürich, Schweiz

Bernhard.Uehleke@usz.ch 
schnittsstudien, das man nur durch prospektiv randomisierte Studien (mit Zuweisung der Patienten über eine Zentrale) ausgleichen könnte. Ein etwas nebenbei erwähnter wichtiger Punkt ist, dass Überweisungen und entsprechende Folgekosten sowie Klinikkosten überhaupt nicht patientenspezifisch erfasst wurden, sondern nur die auf die jeweils untersuchte Praxis bezogenen Kosten. Es könnte damit gut sein, dass Patienten neben den Kosten beim Homöopathen noch zusätzlich weitere Kosten bei anderen Ärzten erzeugen. Somit ist die Studie wenig aussagekräftig. Es zeigt sich aber, dass CAM-Verfahren differenziert betrachtet werden sollten. In der Diskussion erwähnen die Autoren, dass sich ein erheblich kleinerer Anteil von CAM-Ärzten als primäre Behandler einordnet. Patienten der
CAM-Ärzte hatten dementsprechend häufiger chronische und schwerere Erkrankungen. Möglicherweise könnte damit der Kosten/Nutzen-Aspekt für CAM-Verfahren noch günstiger ausfallen. Den Schlussfolgerungen der Autoren, dass CAM Einsparpotenzial biete, kann dennoch nicht uneingeschränkt gefolgt werden. 